\title{
Psychological Resilience Moderates the Effect of Perceived Stress on Late-Life Depression in Community-Dwelling Older Adults
}

\author{
Leandro da Silva-Sauer ${ }^{1}$ (D) - Thiago Regis Gouveia Lima ${ }^{1}$ • \\ Égina Karoline Gonçalves da Fonsêca ${ }^{1}$ - Alejandro de la Torre-Luque ${ }^{2}$. \\ Xiaoqian $\mathrm{Yu}^{3} \cdot$ Bernardino Fernández-Calvo $^{1,4}$ (ID)
}

Accepted: 16 February 2021/Published online: 06 April 2021

(C) Associação Brasileira de Psicologia 2021, corrected publication 2022

\begin{abstract}
Depression is a disabling mental condition that reduces the quality of life regardless of age and circumstances. Late-life depression may be especially impairing due to its relationship with poor physical and mental health. Repeated or prolonged exposures to stressful events deserve a particular interest among late-life depression risk factors. One factor that may help to cope with these stressful situations is the resilience. The objective of the study was to examine the moderating effect of resilience on the relationship between perceived stress (PS) and depression. A total of 1020 community-dwelling older adults aged from 60 to 101 years $(M=68.5, S D=6.99)$ completed the Perceived Stress Scale, Hospital Anxiety and Depression Scale, and Brief Resilience Scale. A moderation effect has been tested using PROCESS for SPSS. Depressive symptomatology was positively related to PS $(r=.598 ; p$ $<.001)$ and inversely related to resilience $(r=-.444 ; p<.001)$. Moreover, the negative impact of PS on depressive symptoms was buffered for individuals with higher resilience $(\beta$ $=-.014 ; p<.001)$. The resilience could be an adaptive strategy to cope with stress and reduce depression in community-dwelling older adults.
\end{abstract}

Keywords Resilience $\cdot$ Protective factor $\cdot$ Perceived stress $\cdot$ Bounce back $\cdot$ Late-life depression - Older adults

\section{Introduction}

The older population has rapidly grown worldwide. By 2050, the number of people over 60 is estimated to reach 2 billion, representing $22 \%$ of the global population

Leandro da Silva-Sauer

dasilvasauer@mail.usf.edu

Bernardino Fernández-Calvo

bfcalvo@uco.es

Extended author information available on the last page of the article 
(World Health Organization and Unicef, 2014). According to data from IBGE (Brazilian Institute of Geography and Statistics), Brazil currently has a population of 20.6 million in this group, and by the year 2060, the country will reach about 58.4 million people in the old age. Considering the numerous social, economic, and physical changes the aging leads to, this scenario alerts to the need for public policies that promote health and life quality for the older adult population (Ávila et al., 2016).

As one of the most prevalent psychiatric disorders among the old age, late-life depression (LLD) negatively affects one's functionality, cognition, and well-being (Alexopoulos, 2019). Besides, frequent relapse and recurrence of LLD are associated with increased mortality and can also be harder to treat compared to younger persons (Haigh et al., 2018). Currently, in Brazil, the estimated average prevalence of depressive symptoms for older residents in the community is $21 \%$ (Meneguci et al., 2019). The high prevalence rate and the adversities are associated with this disease and reinforce the need for immediate attention and development of the interventions to avoid LLD (Portellano-Ortiz et al., 2018).

Numerous studies have supported the relationship between stress and depression. Kendler et al. (1999) showed that stressful life events (SLE) were a significant risk factor of major depression episode onset. In this regard, having experienced a stressful life event in the last month had major depression 5.64 times more likely to be developed. A possible pathophysiological mechanism involved in such a precipitating loading could be that stressful life events may initiate a cascade of alterations in stress response (Yang et al., 2015). These alterations may precipitate behavioral, cognitive, and mood disorders to be manifested ( $\mathrm{Li}$ et al. 2008). On the other hand, chronic stress leads to elevated hormones (e.g., cortisol), and reduced serotonin and other neurotransmitters in the brain, including dopamine, which has been linked to the development of depression (Chaudhury et al., 2013). Therefore, it is not surprising that the extent of exposure to SLE is positively correlated with the development of LLD (Ezzati et al., 2014).

Subjective stress may emerge as a result of a perceived imbalance between environmental demands and individual's coping resources (Lazarus \& Folkman, 1984). Hamarat et al. (2001) found that older adults often report lower levels of perceived stress than younger adults when confronted a SLE. The authors suggested that this could be due to the reluctance of older adults to view situations as stressful. This explanation goes in line with findings from previous studies. For example, Klinger and Spaulding (1998) found that older adults tended to underreport pain or act on it not as readily as younger people. Thus, we can say that, in older people, stress would be better measured as a subjective stress; it means by the perception of stress intensity given to an event to define how this stressful event will affect a person (for more information, see review by Fiske et al., 2009).

In view of the need to reduce stress and prevent it from supporting the development of symptoms of LLD, it is necessary to disentangle specific mechanisms involved in this process aiming at promoting mental health outcomes in older people. The review by Krishnan and Nestler (2008) about the neurobiological bases of depression suggests that the understanding of resilience mechanisms to cope with stress may open new lines of research for the development of effective treatments for depression.

Resilience has been proposed as a protective factor in face of adversity in older adults (Bolton et al., 2016) and against perceived stress or the development of LLD 
(Färber \& Rosendahl, 2020; Laird et al., 2019). However, the literature on the relationship between resilience and LLD is still scarce (Ávila et al., 2016). Resilience is usually thought of as a dynamic process, rather than an individual characteristic (Kalisch et al., 2017), though it is approached as an individual capacity and resource remains common in the literature. Therefore, resilience is a multidimensional construct that has been facing challenges in its definition and measurement over the last decades (Chmitorz et al., 2018). It has been conceptualized in different ways including the ability to recover from stress (Smith et al., 2008; Smith et al., 2010), adapting positively to stressful circumstances or difficulties (Luthar et al., 2000), not getting sick or maintaining stability despite stress (Bonanno, 2004), and functioning above the norm, despite stress or adversity (Tusaie \& Dyer, 2004).

Smith et al. (2008) proposed the Brief Resilience Scale (BRS) to evaluate the resilience as an individual's capacity to withstand stress, which may result in the individual "bouncing back" to a previous state of normal functioning, or simply not showing negative effects. From this standpoint, resilience does not mean avoiding the stressful event, but learn new ways of coping after confrontation. Windle et al. (2011) conducted an extensive review about resilience scales and concluded that the BRS was among the best instruments for assessing resilience due to its excellent psychometric properties. The authors suggested that the BRS might be the only instrument measuring the individual's stress-recovery ability in comparison to the rest of instruments which were more focused on assessing health-related outcomes (e.g., the individual's ability to face a stressful situation and how prepared one would be to deal with stress). This scale has recently been validated with an extensive sample of older Brazilian adults showing excellent psychometric properties (da Silva-Sauer et al., 2020a). This may contribute to gain insight into the potential role of the individual bouncing back ability in the pervasive relationship between stress and depression, because a valid and reliable tool is now available. Although its concept proposes to assess the ability to recover from stress, the definition of resilience proposed by Smith and colleagues (2008) still lacks evidence to understand how this individual resource would work in the relationship between stress and depression, specifically in old age, since there is not a vast literature on the subject.

Previous studies have shown that resilience is associated with a lower reactivity to stress in older adults (Ong et al., 2006); greater satisfaction with life and happiness (Smith \& Hollinger-Smith, 2015); lower rates of depression (Musliner et al., 2015); higher self-rated successful aging and lower levels of perceived stress and depression (da Silva-Sauer, Rodrigues, et al., 2020b); and lower depressive symptoms associated with stressful life events (Jeon et al., 2017; Lim et al., 2015). However, other authors failed to find modulating effects (moderation or mediation) between resilience and perceived stress, suggesting that resilient individuals emerged from stressful situations relatively unchanged (Caltabiano \& Caltabiano, 2006; de Paula Couto et al., 2011). The inconsistent results observed across studies may be derived from conceptual and methodological aspects. On the other hand, to our best knowledge, no study has been conducted so far to examine the moderating role of resilience in the impact of perceived stress on depressive symptomatology in older Brazilian adults. 
From a theoretical point of view, the model proposed by the study is justified, since the concept of resilience adopted is the ability to recover from stress, so it would work as the moderator between the perceived stress and the presence of depressive symptoms. In this study, we analyzed the dispositional trait of resilience in older Brazilian adults, conceptualized as the ability to recover from stress (Smith et al., 2008). Additionally, stress was conceptualized as the subjective interpretation of SLE. Thus, this study aimed to provide some insight into the role of resilience in the relationship between perceived stress and depressive symptoms among older Brazilians. More specifically, we were interested in examining (1) the relationship between resilience and depressive symptoms in community-dwelling older adults facing stressful life events, and (2) the moderating role of resilience on the relationship between perceived stress and depressive symptoms among community-dwelling seniors. We hypothesized that there will be positive relationship between perceived stress scores and depressive symptoms; and resilience moderates this association in a way that higher level of resilience reduces depressive symptoms even with high perceived stress scores.

\section{Methods}

\section{Participants}

Participants were 1020 older adults aged 60 to 101 years $(M=68.50 ; S D=6.99)$. Other descriptive data for the sample can be seen in Table 1. The inclusion criteria were as follows: (1) age is or above 60 years, which follows the criteria established by the World Health Organization to characterize older adult in undeveloped countries; and (2) ability to understand and answer questions, in either written (literate) or verbal (not literate) form, and without history of dementia.

\section{Measures}

\section{Demographics}

Demographic information including age, gender (female or male), marital status (married or other), and education were collected.

\section{Resilience}

Brief Resilience Scale (BRS; Smith et al., 2008) The BRS was a 6-item questionnaire used to assess an individual's ability to recover from a stressful event. The participants indicated the degree of agreement on a Likert scale ranging from 1 (strongly disagree) to 5 (strongly agree). Total scores are averaged, ranging from 1 to 5 , and higher scores indicate a greater level of resilience. The Brazilian version of the BRS (BRS-B) was validated by da Silva-Sauer et al. (2020a). Examples of statements from the scale in the English version are the following: "I tend to bounce back quickly after hard times"; "It does not take me long to recover from a stressful event"; "I usually come through difficult times with little trouble." 
Table 1 The descriptive statistics of the outcome measures used according to the sociodemographic characteristics of the participants

\begin{tabular}{|c|c|c|c|c|c|}
\hline \multicolumn{2}{|l|}{ Variable } & \multirow{2}{*}{$\begin{array}{l}N \\
433\end{array}$} & \multirow{2}{*}{$\begin{array}{l}\text { PSS-10 } \\
M(S D) \\
13.5(6.7)\end{array}$} & \multirow{2}{*}{$\begin{array}{l}\text { BRS } \\
M(S D) \\
20.2(4.2)\end{array}$} & \multirow{2}{*}{$\begin{array}{l}\text { HADS-D } \\
M(S D) \\
5.5(3.7)\end{array}$} \\
\hline Sex & Male & & & & \\
\hline & Female & 587 & $14.5(6.8)$ & 19.9(3.9) & $5.3(3.6)$ \\
\hline & Total & 1020 & $14.0(6.8)$ & $20.1(4.0)$ & $5.4(3.7)$ \\
\hline \multirow[t]{4}{*}{ Race } & White & 482 & $13.8(7.3)$ & $20.3(4.3)$ & $5.1(3.8)$ \\
\hline & Black & 116 & $14.9(6.4)$ & $19.4(4.0)$ & $5.8(3.5)$ \\
\hline & Grayish-brown & 417 & $14.0(6.3)$ & $19.9(3.8)$ & $5.6(3.5)$ \\
\hline & Total & 1015 & $14.0(6.8)$ & $20.1(4.1)$ & $5.4(3.6)$ \\
\hline \multirow[t]{5}{*}{ Education } & Basic education & 396 & $15.3(6.9)$ & 19.1(4.1) & $6.1(3.7)$ \\
\hline & Elementary education & 383 & $13.5(6.8)$ & $20.2(4.1)$ & $5.2(3.7)$ \\
\hline & High school & 125 & $13.5(6.2)$ & $20.4(3.5)$ & $4.4(3.4)$ \\
\hline & College education & 114 & $11.9(5.7)$ & $22.7(3.3)$ & $4.3(3.0)$ \\
\hline & Total & 1018 & $14.0(6.8)$ & $20.1(4.1)$ & $5.4(3.6)$ \\
\hline \multirow[t]{5}{*}{ Marital status } & Single & 125 & $14.6(5.7)$ & $20.0(3.9)$ & $5.8(3.2)$ \\
\hline & Married & 468 & $13.3(6.9)$ & $20.2(4.2)$ & $5.2(3.7)$ \\
\hline & Widower & 223 & $15.0(6.6)$ & $19.8(4.0)$ & $5.5(3.6)$ \\
\hline & Divorced/separated & 176 & $14.7(7.0)$ & $20.0(3.9)$ & $5.7(4.0)$ \\
\hline & Total & 992 & $14.1(6.7)$ & $20.0(4.1)$ & $5.4(3.6)$ \\
\hline
\end{tabular}

$M(S D)$ : mean and standard deviation; PSS-10: Perceived Stress Scal; BRS: Brief Resilience Scale; HADS-D: depression subscale of the Hospital Anxiety and Depression Scale

Basic education: up to 6 years of education; elementary education: from 7 to 12 years of education; high school: from 13 to 16 years of education; college education: over 17 years of education. Totals for race, participants, and civil status do not reach 1020 (the whole sample) because some participants did not answer this question

\section{Perceived Stress}

Perceived Stress Scale (PSS-10; Cohen et al., 1983) The scale measured overall perceived stress experienced across the past 30 days on a 5-point Likert scale from 0 (never) to 4 (always). The PSS-10 total scores range from 0 to 40, with higher scores reflecting greater perceived stress. The Brazilian version of the PSS-10 scale was used in the current study (Luft et al., 2007). Examples of questions from the scale in the English version are the following: "In the last month, how often have you felt difficulties were piling up so high that you could not overcome them?"; "In the last month, how often have you felt nervous and stressed?"; "In the last month, how often have you felt that you were unable to control the important things in your life?"

\section{Depressive Symptomatology}

The Hospital Anxiety and Depression Scale (HADS; Zigmond \& Snaith, 1983) was used to measure psychological distress. The self-report is composed of 14 items and consists of two subscales with 7 items each: anxiety (HADS-A) and depression (HADS-D). Each statement 
is rated on a 4-point Likert response format ranging from 0 to 3 , reaching a total score from 0 to 21 for both scales. The Brazilian version of the HADS has been validated by Botega et al. (1995), but we only considered the HADS-D scale in this study. Higher scores indicate a higher level of depressive symptomatology. Examples of statements from the scale in the English version are the following: "I feel as if I am slowed down"; "I can laugh and see the funny side of things"; "I have lost interest in my appearance."

\section{Procedure}

The cross-sectional study was approved by the Research Ethics Committee of the University Hospital Júlio Müller (106 / CEP - HUJM / 2011). Participation was voluntary, with each participant signing the informed consent terms, duly drafted according to resolution $\mathrm{N}^{\circ} 466 / 12$ of the Brazilian National Health Council. The conceptual framework of this study can be seen in Fig. 1.

the objectives, procedures, data reliability, and anonymity of their participation. The researchers subsequently verified the ages of the interviewees and the self-reports were completed voluntarily. A researcher assisted the participant in case they were unable to either read or write. The application time of the instruments was between 5 and $7 \mathrm{~min}$, and the subsequent order sociodemographic questionnaire, BRS, PSS-10, and HADSD was followed.

\section{Data Analysis}

Descriptive and inferential statistics (mean and standard deviation) were calculated for all sociodemographic data and scores for each scale. Pearson's correlation was conducted to assess the relationships between resilience, perceived stress, and depression. McDonald's omega coefficient ( $\omega$ ) (McDonald, 1999) was calculated to test the internal consistency reliability following suggestions made by Hayes and Coutts (2020) with the OMEGA macro.

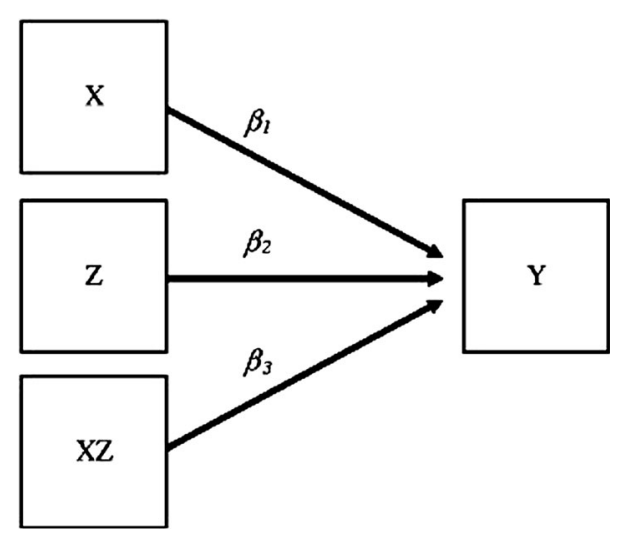

Fig. 1 Conceptual framework. Moderation model. $X=$ the independent variable, that is, scores on the PSS scale of perceived stress; $Y=$ the dependent variable, that is, scores on the HADS scale of depression; $Z=$ the moderating variable, scores on the BRS resilience scale; $X Z=$ this interaction represents the product of $X$ and the moderating variable; $\beta 1=$ the effect of $X$ on $Y ; \beta 2=$ the effect of $Z$ on $Y ; \beta 3=$ the effect of $X Z$ on $Y$. The results can be seen in Table 3 
Moderation analysis was conducted to test the relationship between perceived stress (PPS-10) and depressive symptomatology (HASD-D), as moderated by resilience (BRS), using PROCESS macro, version.3.5 (Hayes, 2018), for Statistical Package for Social Sciences (SPSS), which follows procedures recommended by Hayes (2018) to test for moderation. A moderation model was verified with 10,000 bootstrap samples, with $95 \%$ confidence interval, and with continuous variables that define product meancentered. Participants' PSS-10 scores were entered into the model as the independent variable $(X)$, BRS scores as the moderator $(M)$, and HADS-D scores as the dependent variable $(Y)$ with age, gender, education, and marital status included as covariates. Linearity assumptions, normally distributed errors, and uncorrelated errors were verified and met. Simple slope tests were used to determine the focal predictor effect on dependent variable at three levels of the moderator (low, medium, and high), corresponding at the 16th, 50th, and 84th percentiles, since the moderator was normally distributed. A plot was made with the low, medium, and high values for focal predictor and moderator variables in order to make the interpretation clearer. Finally, the Johnson-Neyman procedure was used to check regions of significance for the examined moderator effect. Analyses were carried out using SPSS, version 25 (IBM Corporation, Armonk, NY, USA), and $p$ values $<.05$ were considered statistically significant.

\section{Results}

Table 1 displays the results in each of the outcome measures used according to the sociodemographic characteristics of participants. Table 2 shows the internal consistency reliability, mean, standard deviation, and correlation between the outcome measures used in the study. All measures presented adequate internal consistency. Depressive symptomatology was positively associated to perceived stress and negatively related to resilience, while perceived stress and resilience were also negatively correlated.

\section{A Simple Moderation Model}

Table 3 displays the moderating role of resilience on the relationship between perceived stress and depressive symptomatology. There was a significant association between perceived stress and depression symptoms, controlling for age, gender, education, and

Table 2 Correlations and descriptive analysis of outcome measures

\begin{tabular}{|c|c|c|c|c|c|c|c|}
\hline & Scales & 2 & 3 & Mean $(S D)$ & Min & Max & $\omega$ \\
\hline 1 & BRS & $-.479 * *$ & $-.444 * *$ & $20.10(4.11)$ & 06 & 30 & .680 \\
\hline 2 & PSS-10 & & $.598 * *$ & $14.07(6.82)$ & 00 & 39 & .830 \\
\hline 3 & HADS-D & & & $5.43(3.68)$ & 00 & 19 & .770 \\
\hline
\end{tabular}

***earson correlation is significant at the 0.001 level (2-tailed)

$N=1020 ;$ BRS: Brief Resilience Scale; PSS-10: Perceived Stress Scale; HADS-D: depression subscale of the Hospital Anxiety and Depression Scale; Mean (SD) showed the mean punctuation and the standard deviation to each scale; Min is the minimum score in the scale; Max is the maximum score; $\omega$ : McDonald's omega coefficient to each scale 
older adults' marital status. Furthermore, it shows that the interaction term of perceived stress and resilience was significant, confirming that older adults' resilience level moderated the link between perceived stress and their depressive symptoms $\left(R^{2}=\right.$ $.410, F(7,984)=97.73, p<.001)$.

Simple slope analyses showed that older adults' resilience level significantly buffered the effect of perceived stress on depressive symptomatology. As can be seen in Fig. 2, the negative perceived stress effect appears to be larger among the relatively non-resilient (low resilience; $b=.33$, IC 95\% [.29, .37], $t=17.13, p<.001$ ) than among the relatively resilient counterparts (high resilience; $b=.214$, IC 95\% [.18, .25], $t=$ $11.18, p<.001)$. Additionally, there were no points within the observed value range of the moderator (resilience) where the conditional effect of perceived stress on depressive symptoms transits between statistically significant and not significant, according to the Johnson-Neyman method (see Fig. 3). As can be seen in this plot, the conditional effect of the perceived stress on depressive symptomatology as a function of resilience was negative; that is, as the resilience increases, the effect of perceived stress in the presence of depressive symptoms decreases.

\section{Discussion}

The study examined the moderating role of resilience, as the ability to bounce back from stress, on the relationship between perceived stress and depressive symptomatology in a large sample of Brazilians older adults. Our results supported the hypothesis that resilience acts as a protective factor against depressive symptoms among older adults although this effect seems to depend on the intensity of the perceived stress. Also, the results showed that greater intensity of perceived stress was associated with higher scores on the presence of depressive symptoms, independent of the levels of resilience. However, for resilience scores, the BRS tends to represent a mitigating factor that helps to understand how often, and how much, perceived stress will trigger depressive symptoms among older adults.

Our results are in line with previous studies that reported a moderate negative correlation of resilience with both perceived stress and depression (Ávila et al., 2016; da Silva-Sauer et al., 2020, b; Ong et al., 2006), suggesting that older adults who are more resilient tend to perceive daily stressful situations and psychological distress less stressful than older adults who are less resilient.

Table 3 Multiple regression analysis summary for depressive symptomatology and perceived stress moderated by resilience $(N=1020)$

\begin{tabular}{lllll}
\hline Independent variables & $b[\mathrm{LLCI}, \mathrm{ULCI}]$ & $\mathrm{SE}$ & $t$ & $p$ \\
\hline Resilience (centered) & $-.169[-.219,-.120]$ & .025 & -6.68 & .001 \\
Perceived stress (centered) & $.269[.239, .299]$ & .015 & 17.66 & .001 \\
Perceived stress $\times$ resilience & $-.014[-.020,-.008]$ & .002 & -4.88 & .001 \\
Constant & $6.07[3.92,8.22]$ & 1.09 & 5.54 & .001 \\
\hline
\end{tabular}

$R^{2}=.408, F[7,984]=97.73, p<.001 ; \Delta R^{2}=.014, \Delta F(1,984)=23.87, p<.001, b=-.014, t(984)=-4.88$, $p<.001 ; N=1020 . b$ unstandardized beta, SE standard error; $L L C I$ and $U L C I$ lower and upper levels for confidence interval, respectively. Dependent variable was depressive symptoms 


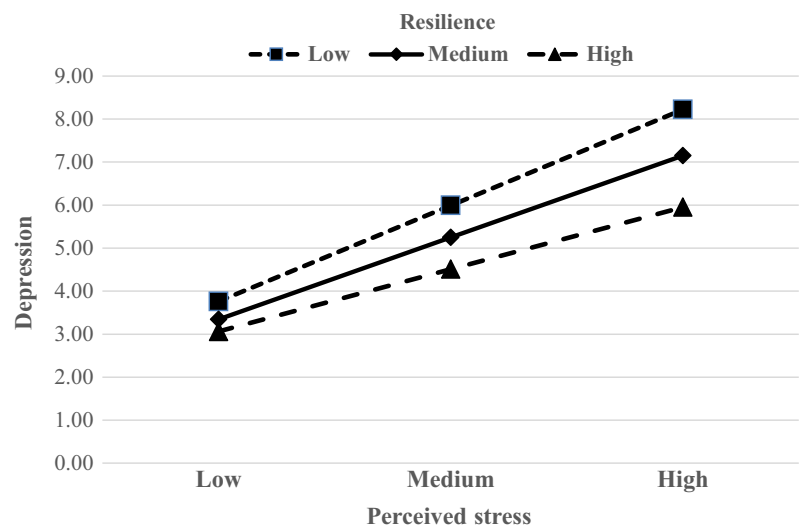

Fig. 2 Simple slope equations of the regression of perceived stress on depressive symptoms at three levels of resilience. The $x$-axis shows the levels of the perceived stress scale (PSS): low (1), medium (2), and high (3). The $y$-axis shows the HADS-D scores. Simple slope for the medium level of the moderating variable: $(b=.27$, IC $95 \%[.24, .30], t=17.74, p<.001)$

In analyses considering the cutoff scores of the scales, it is suggested that, in people with low levels of stress, the effect on depression does not seem to vary by levels of resilience. Among people with high perceived stress ratings, high resilience ratings seem to attenuate the likelihood of developing depression, since depression scores remained below the cutoff point with the presence of moderate depressive symptoms (HADS-D equal or $<7$; Zigmond \& Snaith, 1983) in these individuals, indicating that resilience acts as a protective factor to reduce the impact of stress on the development of depression among older adults with high stress. This effect attenuates in low resilience levels.

While our findings on the effect of resilience as a moderator are consistent with the findings reported in Lim et al. (2015), two main differences in methodology should be noted. First, Lim et al. (2015) used a method that quantifies specific life events (e.g., loss of family members) in a defined period as a cumulative stress score. Such values

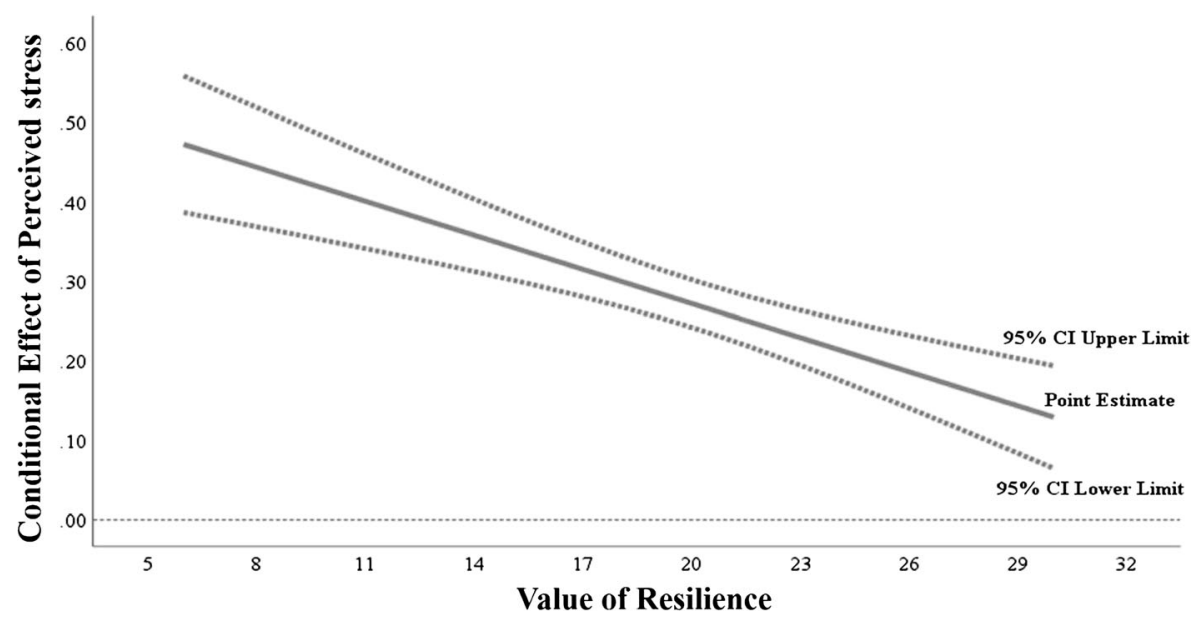

Fig. 3 The conditional effect of the perceived stress on depressive symptomatology as a function of resilience 
are usually based on the number of events, or a sum of weighted events to reflect the adjustment difficulties during the determined time. This effect is tested by García-León et al. (2019), who provided the first data on stressful events (number and intensity) exploring their relationship with resilience. The authors found a similar effect size between the high and low resilient groups in the number and intensity of current stressful events. At this point, our results using a measure of stress intensity showed results of the moderating effect similar to those found by Lim et al. (2015) through the stress measured by the number of events.

Second, we assessed resilience due to the assumption/theory that older adults may develop resilience toward stressful events because they have had extended exposure to life stressful events, such as losses and diseases (Cheetham-Blake et al., 2019; Smith \& Hollinger-Smith, 2015). Therefore, it would be useful to assess resilience as the recovering capacity from stress among older people. In Smith et al. (2010), the authors proposed that the ability to bounce back from stress is prospectively related to better health outcomes following stress. In the review of resilience scales by Windle et al. (2011), the BRS aims to assess resilience as an outcome, that is, the ability to "bounce back", while the resilience scale, used in Lim et al. (2015), was developed for clinical practice as a measure of stress coping ability. Both instruments received the best psychometric ratings among the revised resilience scales. This subtle difference between the proposed measures could have different effects on moderating resilience, which was not observed. The similar effect between the two measures of resilience could be explained by the way the stressors and resilience are linked. Oken et al. (2015) propose this relation as a dynamic system. In other words, it would not be the prolonged exposure, but the way people face previously stressful experiences that will determine if they will succeed in managing future stressful situations.

Another point is the difference between the cultures of the older people who comprise the sample of the studies. Previous studies reported differences between Asian older adults when compared to older adults from Western cultures (Giles et al., 2003; Löckenhoff et al., 2009). In this sense, the present study corroborates the findings of Lim et al. (2015); even if the differences in the measurement instruments and the sample of older community members of a country with Western customs are considered, the moderating effect of resilience in the relationship between stress and depression seems to be the same as found in the Asian sample.

Resilience to stressful situations is not an uncommon trait and most people display some degree of it; however, stressful life events can overload these mechanisms (Laird et al., 2019). Resilience interventions have been proposed with a focus on expanding specific skills or abilities such as the ability to bounce back. In this sense, resilience seems to really act in the adaptation to stressful circumstances, preventing the development of depressive symptoms. Therefore, if an individual can develop appropriate coping strategies, this will facilitate adaptation and the successful recovery from stressful events in adult life (Wilcox et al., 2003). Understanding these risks and the factors that promote successful adaptation is considered essential to increase resilience and decrease the adverse effects of stress, thus preventing the onset of depressive symptoms. In a recent study, Kimhi et al. (2020) also showed that individual resilience is one of the psychological attributes that can help reduce the impact of the level of distress and sense of danger symptoms of the COVID-19 pandemic. Likewise, our findings showed that the moderator effect of resilience in older adults' mental health 
suggests that the ability to handle stressful situation such as the current COVID-19 pandemic scenario or a future catastrophic event will be strengthened by interventions that focus on the cultivation of positive constructs including resilience, and other factors strongly linked to it, such as optimism, sense of control and coherence, self-compassion, self-efficacy, and self-esteem (Bonanno, 2004; Windle et al., 2011).

\section{Limitations}

Several limitations must to be noted. First, these findings are based on a confirmatory cross-sectional model; therefore, future research should investigate the longitudinal effect to understand how the moderation effect would be presented over time. Second, in this study, we consider resilience only as an individual characteristic, disregarding the social support that one may receive. In this sense, we suggest future studies to assess the effect of interpersonal resources of resilience on the relationship between stress and depression. Moreover, there were sensitive items participants may not honestly report in the presence of researchers. Although we use validated measures widely used by the scientific community, it is considered that, due to the complexity of the evaluated constructs, the instruments used in this study may not be the most appropriate. For example, the HADS-D is a screening tool for depressive symptoms that does not guarantee a diagnosis of depression. Finally, our results were focused on exploring the subjective dimension of resilience, as a trait. In this sense, the BRS measures the dispositional ability to bounce back, but not how the individual may recover after confronting a specific stressful situation. Further studies should cover this gap by adding evidence from other resilience conceptualizations of individual's resilience (redundante. Sugestão: apagar o primeiro resilience). Despite the limitations, our findings provide a basis for future studies to develop models to investigate the role of resilience as a protective factor between stress and LLD.

\section{Conclusion}

Resilience as the capacity to bounce back from stress has a positive impact on psychological health and an exceptional place on behavioral research. Our results suggest that older adults with high resilience experience less depressive symptoms during stressful situations. The greatest effect of resilience seems to occur when stressful situations are perceived as more intense. These findings help explain how resilience works in older adults and reinforce the protective role of resilience against LLD, which is the leading cause of disability in this group. Given the rapid aging of population, it is important to develop interventions that may increase resilience in older adults to improve their ability to cope with stress and promote psychological health.

Funding Preparation of this article was supported by the Beatriz Galindo Programme of General Secretary of Universities at the Ministry of Science, Innovation, and Universities (ref. BEAGAL18/00006) and the National Brazilian National Council of Technological and Scientific Development in Brazil 307366/2017-0 to BFC. 


\section{Declarations}

Conflict of Interest The authors declare no competing interests.

\section{References}

Alexopoulos, G. S. (2019). Mechanisms and treatment of late-life depression. Translational Psychiatry, 9, 188. https://doi.org/10.1038/s41398-019-0514-6.

Ávila, M. P. W., Lucchetti, A. L. G., \& Lucchetti, G. (2016). Association between depression and resilience in older adults: A systematic review and meta-analysis. International Journal of Geriatric Psychiatry, 32(3), 237-246. https://doi.org/10.1002/gps.4619.

Bolton, K. W., Praetorius, R. T., \& Smith-Osborne, A. (2016). Resilience protective factors in an older adult population: A qualitative interpretive meta-synthesis. Social Work Research, 40(3), 171-182. https://doi. org/10.1093/swr/svw008.

Bonanno, G. A. (2004). Loss, trauma, and human resilience. American Psychologist, 59, 20-28. https://doi. org/10.1037/0003-066X.59.1.20.

Botega, N. J., Bio, M. R., Zomignani, M. A., Garcia Jr., C., \& Pereira, W. A. (1995). Mood disorders among inpatients in ambulatory and validation of the anxiety and depression scale HAD. Revista de Saúde Pública, 29, 355-363.

Caltabiano, Marie L., and Caltabiano, Nerina J. (2006). Resilience and health outcomes in the elderly. In: Proceedings of the 39th Annual Conference of the Australian Association of Gerontology., November 22-24, 2006. Sydney, NSW, Australia. Available at: http://eprints.jcu.edu.au/4271.

Chaudhury, D., Walsh, J. J., Friedman, A. K., Juarez, B., Ku, S. M., Koo, ..., \& Han, M. H. (2013). Rapid regulation of depression-related behaviours by control of midbrain dopamine neurons. Nature, 493(7433), 532-536. https://doi.org/10.1038/nature11713

Cheetham-Blake, T. J., Turner-Cobb, J. M., Family, H. E., \& Turner, J. E. (2019). Resilience characteristics and prior life stress determine anticipatory response to acute social stress in children aged 7-11 years. British Journal of Health Psychology, 24(2), 282-297. https://doi.org/10.1111/bjhp.12353.

Chmitorz, A., Kunzler, A., Helmreich, I., Tüscher, O., Kalisch, R., Kubiak, T., Wessa, M., \& Lieb, K. (2018). Intervention studies to foster resilience - A systematic review and proposal for a resilience framework in future intervention studies. Clinical Psychology Review, 59, 78-100. https://doi.org/10.1016/j.cpr.2017. 11.002 .

Cohen, S., Karmack, T., \& Mermelsteinm, R. A. (1983). Global measure of perceived stress. Journal of Health and Social Behavior, 24(4), 385-396. https://doi.org/10.1590/1413-81232015205.00502014.

da Silva-Sauer, L., de la Torre-Luque, L., Smith, B. W., Lins, M. C., Andrade, S., \& Fernández-Calvo, B. (2020a). Brief Resilience Scale (BRS) Portuguese Version validity and metrics for the older adult population. Aging \& Mental Health, 1-10. https://doi.org/10.1080/13607863.2020.1753015.

da Silva-Sauer, L., Rodrigues, R. M., de la Torre-Luke, A., \& Fernández-Calvo, B. (2020b). Cross-cultural adaptation and psychometric properties of the Brazilian Portuguese version of Successful Aging Scale in community-dwelling older adults. Journal of Community Psychology, 1, 1-13. https://doi.org/10.1002/ jcop. 22374.

de Paula Couto, M. C. P., Koller, S. H., \& Novo, R. (2011). Stressful Life Events and Psychological Wellbeing in a Brazilian Sample of Older Persons: The Role of Resilience. Ageing International, 36(4), 492505. https://doi.org/10.1007/s12126-011-9123-2

Ezzati, A., Jiang, J., Katz, M. J., Sliwinski, M. J., Zimmerman, M. E., \& Lipton, R. B. (2014). Validation of the Perceived Stress Scale in a community sample of older adults. International Journal of Geriatric Psychiatry, 29, 645-652. https://doi.org/10.1002/gps.4049.

Färber, F., \& Rosendahl, J. (2020). Trait resilience and mental health in older adults: A meta-analytic review. Personality and Mental Health, 14, 361-375. https://doi.org/10.1002/pmh.1490.

Fiske, A., Wetherell, J. L., \& Gatz, M. (2009). Depression in older adults. Annual Review of Clinical Psychology, 5(1), 363-389. https://doi.org/10.1146/annurev.clinpsy.032408.153621.

García-León, M. Á., Pérez-Mármol, J. M., Gonzalez-Pérez, R., García-Ríos, M. D. C., \& Peralta-Ramírez, M. I. (2019). Relationship between resilience and stress: Perceived stress, stressful life events, HPA axis response during a stressful task and hair cortisol. Physiology \& Behavior, 202, 87-93. https://doi.org/10. 1016/j.physbeh.2019.02.001. 
Giles, H., Noels, K. A., Williams, A., Ota, H., Lim, T.-S., Ng, S. H., Ryan, E. B., \& Somera, L. (2003). Intergenerational communication across cultures: Young people's perceptions of conversations with family elders, non-family elders and same-age peers. Journal of Cross-Cultural Gerontology, 18, 1-32. https://doi.org/10.1023/A:1024854211638.

Haigh, E. A. P., Bogucki, O. E., Sigmon, S. T., \& Blazer, D. G. (2018). Depression among older adults: A 20year update on five common myths and misconceptions. The American Journal of Geriatric Psychiatry, 26, 107-122. https://doi.org/10.1016/j.jagp.2017.06.011.

Hamarat, E., Thompson, D., Zabrucky, K. M., Steele, D., Matheny, K. B., \& Aysan, F. (2001). Perceived stress and coping resource availability as predictors of life satisfaction in young, middle-aged, and older adults. Experimental Aging Research, 27(2), 181-196. https://doi.org/10.1080/036107301750074051.

Hayes, A. F. (2018). Introduction to mediation, moderation, and conditional process analysis: A regressionbased approach (2nd ed.). The Guilford Press.

Hayes, A. F., \& Coutts, J. J. (2020). Use omega rather than Cronbach's alpha for estimating reliability. Communication Methods and Measures, 14(1), 1-24. https://doi.org/10.1080/19312458.2020.1718629.

Jeon, G.-S., Park, S.-Y., \& Bernstein, K. S. (2017). Socio-demographic and psychological correlates of posttraumatic growth among Korean Americans with a history of traumatic life experiences. Archives of Psychiatric Nursing, 31(3), 256-262. https://doi.org/10.1016/j.apnu.2016.12.002.

Kalisch, R., Baker, D., Basten, U., Boks, M., Bonanno, G., Brummelman, E., et al. (2017). The resilience framework as a strategy to fight stress-related disorders: Current challenges and future perspectives. Nature Human Behaviour, 1(11), 784-790. https://doi.org/10.1038/s41562-017-0200-8.

Kendler, K. S., Karkowski, L. M., \& Prescott, C. A. (1999). Causal relationship between stressful life events and the onset of major depression. The American Journal of Psychiatry, 156(6), 837-841. https://doi.org/ 10.1176/ajp.156.6.837.

Kimhi, S., Marciano, H., Eshel, Y., \& Adini, B. (2020). Recovery from the COVID-19 pandemic: Distress and resilience. International Journal of Disaster Risk Reduction, 50, 101843. https://doi.org/10.1016/j.ijdrr. 2020.101843.

Klinger, L., \& Spaulding, S. J. (1998). Chronic pain in the elderly: Is silence really golden? Physical \& Occupational Therapy in Geriatrics, 15(3), 1-17.

Krishnan, V., \& Nestler, E. J. (2008). The molecular neurobiology of depression. Nature, 455(7215), 894 902. https://doi.org/10.1038/nature07455.

Laird, K. T., Krause, B., Funes, C., \& Lavretsky, H. (2019). Psychobiological factors of resilience and depression in late life. Translational Psychiatry, 9, 88. https://doi.org/10.1038/s41398-019-0424-7.

Lazarus, R., \& Folkman, S. (1984). Stress, appraisal, and coping. Springer.

Li, S., Wang, C., Wang, W., Dong, H., Hou, P., \& Tang, Y. (2008). Chronic mild stress impairs cognition in mice: from brain homeostasis to behavior. Life Sciences, 82, 934-942. https://doi.org/10.1001/archpsyc. 1981.01780290105011.

Lim, M. L., Lim, D., Gwee, X., Nyunt, M. S. Z., Kumar, R., \& Ng, T. P. (2015). Resilience, stressful life events, and depressive symptomatology among older Chinese adults. Aging \& Mental Health, 19(11), 1005-1014. https://doi.org/10.1080/13607863.2014.995591.

Löckenhoff, C. E., de Fruyt, F., Terracciano, A., McCrae, R. R., de Bolle, M., Costa, P. T., Aguilar-Vafaie, M. E., Ahn, C. K., Ahn, H. N., Alcalay, L., Allik, J., Avdeyeva, T. V., Barbaranelli, C., Benet-Martínez, V., Blatný, M., Bratko, D., Cain, T. R., Crawford, J. T., Lima, M. P., Ficková, E., Gheorghiu, M., Halberstadt, J., Hřebíčková, M., Jussim, L., Klinkosz, W., Knežević, G., de Figueroa, N. L., Martin, T. A., Marušić, I., Mastor, K. A., Miramontez, D. R., Nakazato, K., Nansubuga, F., Pramila, V. S., Realo, A., Rolland, J. P., Rossier, J., Schmidt, V., Sekowski, A., Shakespeare-Finch, J., Shimonaka, Y., Simonetti, F., Siuta, J., Smith, P. B., Szmigielska, B., Wang, L., Yamaguchi, M., \& Yik, M. (2009). Perceptions of aging across 26 cultures and their culture-level associates. Psychology and Aging, 24(4), 941-954. https://doi.org/10.1037/a0016901.

Luft, C. D. B., Sanches, S. D. O., Mazo, G. Z., \& Andrade, A. (2007). Versão brasileira da Escala de Estresse Percebido: tradução e validação para idosos. Revista de Saúde Pública, 41, 606-615. https://doi.org/10. 1590/s0034-89102007000400015.

Luthar, S. S., Cicchetti, D., \& Becker, B. (2000). The construct of resilience: A critical evaluation and guidelines for future work. Child Development, 71, 543-562. https://doi.org/10.1111/1467-8624.00164.

McDonald, R. P. (1999). Test theory: A unified treatment. Lawrence Erlbaum.

Meneguci, J., Meneguci, C. A. G., Moreira, M. M., Pereira, K. R., Tribess, S., Sasaki, J. E., \& Virtuoso, J. J. S. (2019). Prevalência de sintomatologia depressiva em idosos brasileiros: uma revisão sistemática com metanálise. Jornal Brasileiro de Psiquiatria, 68(4), 221-230. https://doi.org/10.1590/00472085000000250 . 
Musliner, K. L., Seifuddin, F., Judy, J. A., Pirooznia, M., Goes, F. S., \& Zandi, P. P. (2015). Polygenic risk, stressful life events and depressive symptoms in older adults: A polygenic score analysis. Psychological Medicine, 45(8), 1709-1720. https://doi.org/10.1017/S0033291714002839.

Oken, B. S., Chamine, I., \& Wakeland, W. (2015). A systems approach to stress, stressors and resilience in humans. Behavioural Brain Research, 282, 144-154. https://doi.org/10.1016/j.bbr.2014.12.047.

Ong, A. D., Bergeman, C. S., Bisconti, T. L., \& Wallace, K. A. (2006). Psychological resilience, positive emotions, and successful adaptation to stress in later life. Journal of Personality and Social Psychology, 91(4), 730-749. https://doi.org/10.1037/0022-3514.91.4.730.

Portellano-Ortiz, C., Garre-Olmo, J., Calvó-Perxas, L., \& Conde-Sala, J. L. (2018). Depresión y variables asociadas en personas mayores de 50 años en España. Revista de Psiquiatría y Salud Mental, 11(4), 216226. https://doi.org/10.1016/j.rpsm.2016.10.003.

Smith, B. W., Dalen, J., Wiggins, K., Tooley, E., Christopher, P., \& Bernard, J. (2008). The brief resilience scale: Assessing the ability to bounce back. International Journal of Behavioral Medicine, 15(3), 194 200. https://doi.org/10.1080/10705500802222972.

Smith, J. L., \& Hollinger-Smith, L. (2015). Savoring, resilience, and psychological well-being in older adults. Aging \& Mental Health, 19(3), 192-200. https://doi.org/10.1080/13607863.2014.986647.

Smith, B. W., Tooley, E. M., Christopher, P., \& Kay, V. S. (2010). Resilience as the ability to bounce back from stress: A neglected personal resource? Journal of Positive Psychology, 5(93), 166-176. https://doi. org/10.1080/17439760.2010.482186.

Tusaie, K., \& Dyer, J. (2004). Resilience: A historical review of the construct. Holistic Nursing Practice, 18, 3-8. https://doi.org/10.1097/00004650-200401000-00002.

Wilcox, S., Evenson, K., Aragaki, A., et al. (2003). The effects of widowhood on physical and mental health, health behaviors, and health outcomes: The Women's Health Initiative. Health Psychology, 22, 513-522. https://doi.org/10.1037/0278-6133.22.5.513.

Windle, G., Bennett, K. M., \& Noyes, J. (2011). A methodological review of resilience measurement scales. Health and Quality of Life Outcomes, 9, 8. https://doi.org/10.1186/1477-7525-9-8.

World Health Organization, \& Unicef. (2014). Trends in maternal mortality: 1990 to 2013: Estimates by WHO. UNICEF, UNFPA, The World Bank and the United Nations Population Division.

Yang, L., Zhao, Y., Wang, Y., Liu, L., Zhang, X., Li, B., \& Cui, R. (2015). The effects of psychological stress on depression. Current Neuropharmacology, 13(4), 494-504. https://doi.org/10.2174/ 1570159x1304150831150507.

Zigmond, A. S., \& Snaith, R. P. (1983). The hospital anxiety and depression scale. Acta Psychiatrica Scandinavica, 67(6), 361-370. https://doi.org/10.1111/j.1600-0447.1983.tb09716.x.

\section{Affiliations}

\section{Leandro da Silva-Sauer ${ }^{1}$ - Thiago Regis Gouveia Lima ${ }^{1}$ • Égina Karoline Gonçalves da Fonsêca ${ }^{1}$ - Alejandro de la Torre-Luque ${ }^{2}$ Xiaoqian $\mathrm{Yu}^{3}$. Bernardino Fernández-Calvo ${ }^{1,4}$}

1 Department of Psychology, Federal University of Paraiba, João Pessoa, Brazil

2 Center for Biomedical Research in Mental Health (CIBERSAM), Department of Legal Medicine, Psychiatry and Pathology, Complutense University of Madrid, Madrid, Spain

3 Department of Psychology, University of South Florida, Tampa, FL, USA

4 Department of Psychology, Faculty of Psychology and Educational Sciences, University of Córdoba, Córdoba, Spain 